\title{
Tunable spin currents in a biased Rashba ring
}

\author{
V. Moldoveanu ${ }^{1}$ and B. Tanatar ${ }^{2}$ \\ ${ }^{1}$ National Institute of Materials Physics, P.O. Box MG-7, Bucharest-Magurele, Romania \\ ${ }^{2}$ Department of Physics, Bilkent University, Bilkent, 06800 Ankara, Turkey
}

(Received 10 August 2009; revised manuscript received 25 November 2009; published 19 January 2010)

\begin{abstract}
The effect of Rashba spin-orbit coupling on the spin interference in a noninteracting one-dimensional ring connected to two leads is studied theoretically within the nonequilibrium Greens' function formalism. We compute the charge and spin currents and analyze their Aharonov-Bohm oscillations. The geometry of the system is conveniently described by the angle $\delta$ between the two leads. We show that for $\delta=180^{\circ}$ (i.e., for symmetrically coupled leads), a good filtering of up- or down-spin orientation is obtained around half-integer multiples of $\Phi / \Phi_{0}$. These particular flux values are degeneracy points for clockwise and counterclockwise propagating states, corresponding to the same spin orientation in the local spin frame of the ring. In contrast, for the asymmetric coupling, i.e., $\delta=135^{\circ}$, the filter efficiency is maximum around integer multiples of $\Phi / \Phi_{0}$. The numerical results suggest that the spin filtering is obtained when the clockwise or counterclockwise states interfere destructively. It turns out that the spin filtering regime is stable against variations in the bias applied on the system. The quasiperiodic oscillations of the charge current, as a function of the Rashba strength, are obtained and discussed.
\end{abstract}

DOI: 10.1103/PhysRevB.81.035326

PACS number(s): 73.23.Hk, 85.35.Ds, 85.35.Be, 73.21.La

\section{INTRODUCTION}

Quasi-one-dimensional semiconductor rings are ideal candidates for testing quantum coherence at nanoscale and the recent field of mesoscopic interferometry is up to a good extent devoted to such systems. Traditionally, the quantum interference in the ring is tuned by the perpendicular magnetic field and results in the celebrated Aharonov-Bohm oscillations of the conductance in open rings or of the persistent currents in closed rings. ${ }^{1}$ Recently, it has been realized that the spin-orbit interaction (SOI) offers an alternative way of observing interference effects in quantum rings. ${ }^{2}$ The method is efficient because, on one hand, the SOI can be nowadays controlled by a gate voltage placed in the vicinity of the sample ${ }^{3-5}$ and, on the other hand, the Rashba SOI strength in confined structures is considerably larger than in vacuum. ${ }^{6}$ For a structure subjected to a constant magnetic field along the $z$ axis, the confinement potential restricting the electronic motion to the $x-y$ plane leads to the following expression of the spin-orbit coupling:

$$
H_{s o}=\frac{\alpha}{\hbar}[\sigma \wedge(-i \hbar \nabla-e \mathbf{A})]_{z},
$$

where $\alpha$ is the spin-orbit strength, which can be computed from the $k p$ theory. ${ }^{2}$ The key point is that $\alpha$ contains the band gap, which is considerably larger that the Dirac gap.

Theoretically, it was predicted ${ }^{7}$ that by varying the Rashba parameter $\alpha$, one could control the spin interference because the spin-orbit interaction removes the spin degeneracy and brings in different Aharonov-Casher phases of the wave functions associated to different spin states. Using this idea, Bergsten et $a l^{8,9}$ were able to measure AharonovCasher oscillations in an array of quantum ring arrays.

From the theoretical point of view, the spin interference in Rashba rings was extensively studied both in closed and open geometries. Since an exhaustive account on the previous studies is quite impossible, we shall just briefly review some of them. The persistent currents in quasi-one-dimensional closed rings were studied by Splettstoesser et al. ${ }^{10}$ within a continuous model, special attention being paid to the subtle definition of the spin current in the ring. Using the Landauer formula, Frustaglia and Richter ${ }^{11}$ obtained almost periodic oscillations of the conductance of one-dimensional and two-dimensional rings as a function of the Rashba parameter. Molnár et al. ${ }^{12}$ calculated the transmittance of the ring at finite temperature, emphasizing transitions between maxima and minima in the Aharonov-Bohm oscillations at different values of the Rashba parameter.

Nikolic et al. ${ }^{13}$ calculated both the conductance of manychannel Rashba rings and the spin Hall conductance using the Keldysh technique (see also the recent review ${ }^{14}$ ). Recently, Borunda et al. ${ }^{15}$ investigated the electron and hole rings with strong spin-orbit interaction and emphasized the role of the carrier density, ${ }^{15}$ and Stepanenko et al. ${ }^{16}$ studied the differential conductance of heavy-hole rings in order to discern and compare the contributions of the Rashba and Dresselhaus spin-orbit couplings.

The growing interest in mesoscopic rings with tunable SOI comes also from spintronics, where one needs spinpolarized currents. The natural idea is then to exploit the tunability of the Rashba coupling within the ring in order to suppress (in ideal cases) or to considerably inhibit currents associated to one spin orientation while allowing the other one to escape into the leads. Such a device is called a spin filter. The natural generalization of the spin filter device is the spin splitter, namely, a three lead ring, which is able to deliver a spin-up current in one output lead and a spin-down current in the other lead. As shown by Földi et al. ${ }^{17}$ in the framework of scattering theory, one can find a suitable sequence of parameters for the spin splitter operation.

Most of the theoretical papers on spin filters and splitters address two problems: (i) the energy dependence of the Landauer conductance/magnetoconductance at a given Rashba strength; (ii) the modulation of the ring conductance when varying the Rashba strength. ${ }^{18-22}$ Capozza et al. ${ }^{23}$ investi- 
gated the oscillations of the conductance taking both Aharonov-Bohm and spin-orbit Aharonov-Casher phases into account.

Bellucci and Onorato ${ }^{24}$ emphasized that the spin filtering operation cannot be implemented in homogeneous rings in the absence of a magnetic field; they proposed instead to add a nonmagnetic $\delta$-type barrier in order to break the timereversal symmetry. In a recent paper, Cohen et al. ${ }^{25}$ investigated the possibility to operate molecular rings as spin filters and splitters. In these systems, the Zeeman term seems to be the only way to separate the spins as the Rashba and Dresselhaus are too small.

The main aim of this work is to investigate the spin filtering properties of a Rashha interferometer coupled to two leads and subjected to a finite bias. We compute the spin and charge currents and discuss the Aharonov-Bohm oscillations as a function of magnetic field and Rashba strength. We also investigate the filter efficiency for different locations of the contacts to the leads (i.e., for symmetric or asymmetric coupling). This aspect is important and should be relevant for experiments. As shown by Aeberhard et al. ${ }^{26}$ the effect of the asymmetric coupling to the leads is a partial lifting of the conductance zeros. In this work, we show that the asymmetric coupling strongly influences the efficiency of the spin filter. To calculate the spin filtering properties ring-shaped interferometers, we employ the nonequilibrium GreenKeldysh formalism, which allows the calculation of steadystate currents in the nonlinear regime. We also include the Zeeman coupling and, therefore, our results take into account the dependence of the tilt angle on the local spin frame (if the Zeeman coupling is disregarded, one has a single tilt angle, depending only on the Rashba coupling and on the ring radius).

The present model starts from the Hamiltonian proposed by Meijer et al. ${ }^{27}$ which describes a quasi-one-dimensional ring. This Hamiltonian takes properly into account the confining potential that defines the ring and was extensively used in many studies on the Rashba interference. ${ }^{10,11,13}$ In view of the numerical simulations, we discretize this Hamiltonian by choosing an appropriate number of sites the part of the rings' spectrum that contributes to transport coincides with the same region from the continuous spectrum. Therefore, our results are relevant for the continuous systems as well. As in most other approaches to the spin interference in a Rashba ring, we do not include the effect of the electronelectron interaction; this approximation seems quite reasonable. ${ }^{10}$ However, in the case of rings with embedded or side-coupled dots, ${ }^{28,29}$ the intradot Coulomb interaction must be taken into account.

The rest of the paper is organized as follows. Section II contains the formalism and presents relevant equations for the spin currents and Green functions, Sec. III contains the discussion of the numerical results, while Sec. IV is left to conclusions and open problems.

\section{FORMALISM}

We consider noninteracting electrons moving in a ring of radius $R$, which is subjected to a constant perpendicular mag-

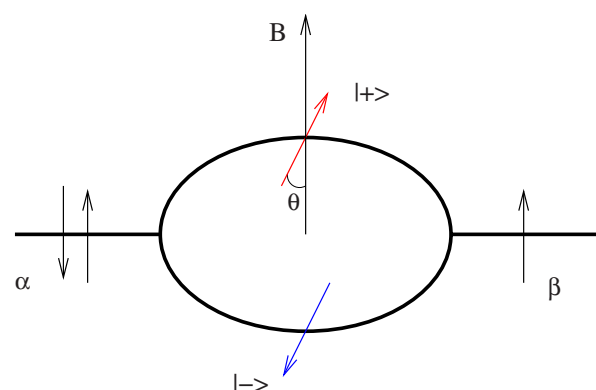

FIG. 1. (Color online) The sketch of the Rashba ring coupled symmetrically to two semi-infinite leads and subjected to a perpendicular magnetic field $B$. There are two spin representations: on the leads, the spin orientation is given by the spinors $|\uparrow, \downarrow\rangle$, while in the ring one should use the proper spinors with respect to the local spin frame defined in the text. We denote them by $|+\rangle$ and $|-\rangle$. For a given orbital quantum number $l$, they are given by Eqs. (5) and (6).

netic field and also coupled to two one-dimensional leads, as shown in Fig. 1. It should be understood that the electrons are forced to move along the ring by a suitable confining potential. Assuming that only the lowest radial subband is important for the transport processes, the Hamiltonian reads as (see Meijer et al. $^{27}$ )

$$
\begin{aligned}
H^{R}= & \frac{\hbar^{2}}{2 m^{*} R^{2}}\left(i \frac{\partial}{\partial \varphi}+\frac{\Phi}{\Phi_{0}}\right)^{2}-\frac{\alpha}{R} \sigma_{r}\left(i \frac{\partial}{\partial \varphi}+\frac{\Phi}{\Phi_{0}}\right)-i \frac{\alpha}{2 R} \sigma_{\varphi} \\
& +\hbar \omega_{z} \sigma_{z}
\end{aligned}
$$

where $\varphi$ is the polar coordinate, $\Phi$ is the flux associated with a constant perpendicular magnetic field $B$, and $\alpha$ is the strength of the Rashba coupling. $\Phi_{0}$ is the unit flux quantum and $\hbar \omega_{z}=g \mu_{B} B$. The matrices $\sigma_{r}$ and $\sigma_{\varphi}$ are defined as usual,

$$
\sigma_{r}=\left(\begin{array}{cc}
0 & e^{-i \varphi} \\
e^{i \varphi} & 0
\end{array}\right), \quad \sigma_{\varphi}=\left(\begin{array}{cc}
0 & -i e^{-i \varphi} \\
i e^{i \varphi} & 0
\end{array}\right) .
$$

The spectrum and eigenfunctions of $H^{R}$ are known and were extensively used in the study of persistent currents in closed rings. ${ }^{10}$ In the transport problem at hand, we shall use a discretized version of the Hamiltonian in Eq. (2); for the simplicity of writing, we use the same notation for the lattice Hamiltonian. We denote by $N$ the number of sites describing the ring, and we define a site-indexed angle $\varphi_{p}=2 \pi p / N$. In the absence of the Rashba coupling, the eigenfunctions of the discretized ring are easily shown to be

$$
\left|\phi_{l}\right\rangle=\frac{1}{\sqrt{N}} \sum_{p=1}^{N} e^{i l \varphi_{p}}|p\rangle
$$

where the orbital quantum number $l=0, \pm 1, \ldots$, $\pm(N / 2-1), N / 2$ (we take $N$ even without loss of generality). Due to the Rashba coupling, one has to introduce a local spin frame characterized by tilt angles $\theta_{l}$. Let $\psi_{l s}$ be the 
eigenfunctions of the ring and $s= \pm 1$ the spin quantum number in the local spin frame. Then one can show by direct calculation that $\left|\psi_{l_{+}}\right\rangle$and $\left|\psi_{l-}\right\rangle$ are given by

$$
\begin{aligned}
& \left|\psi_{l+}\right\rangle=\left(\begin{array}{c}
\cos \left(\frac{\theta_{l}}{2}\right)\left|\phi_{l}\right\rangle \\
\sin \left(\frac{\theta_{l}}{2}\right)\left|\phi_{l+1}\right\rangle
\end{array}\right) \\
& \left|\psi_{l-}\right\rangle=\left(\begin{array}{c}
-\sin \left(\frac{\theta_{l}}{2}\right)\left|\phi_{l}\right\rangle \\
\cos \left(\frac{\theta_{l}}{2}\right)\left|\phi_{l+1}\right\rangle
\end{array}\right),
\end{aligned}
$$

provided that the angle $\theta_{l}$ is constructed such that the offdiagonal elements of $H^{R}$ in the basis $\left\{\psi_{l s}\right\}$ vanish. Straightforward calculations lead to explicit forms for the tilt angle and for the eigenvalues $E_{l s}$ associated to $\left|\psi_{l s}\right\rangle$. As these expressions are rather complicated, we shall not give them here. We have checked that by performing the limit $N \rightarrow \infty$, we recover the expression derived in Ref. 10.

The spectrum and eigenfunctions of the closed ring allow us to write down the spectral representation of the discrete Hamiltonian and the matrix elements of the retarded Green's function,

$$
\begin{gathered}
H^{R}=\sum_{l, s} E_{l s}\left|\psi_{l s}\right\rangle\left\langle\psi_{l s}\right|, \\
g_{p \sigma, p^{\prime} \sigma^{\prime}}^{R}(E)=\sum_{l, s} \frac{\psi_{l s^{*}}(p \sigma) \psi_{l s}\left(p^{\prime} \sigma^{\prime}\right)}{E-E_{l s}+i 0} .
\end{gathered}
$$

In the above equation, $p, p^{\prime}$ are sites along the ring and $\sigma, \sigma^{\prime}=\uparrow, \downarrow$ select up and down components of $\left|\psi_{l s}\right\rangle$, that is, $\psi_{l s}(p \sigma)=\left\langle p \sigma \mid \psi_{l s}\right\rangle$. When the leads are attached to the ring, the quantities one aims to compute are steady-state charge and spin currents. As we shall show later on, the spin filtering properties of the ring depend on the location of the contacts. Therefore, it is useful to write $H^{R}$ in the basis $\{p, \sigma\}$. This can be done using the transformation matrix $\mathbf{R}$ that relates the two bases, i.e., $\left|\psi_{l s}\right\rangle=\sum_{p, \sigma} \mathbf{R}_{p \sigma, l s}|p \sigma\rangle$ with

$$
\mathbf{R}=\left(\begin{array}{cc}
\cos \frac{\theta_{l}}{2} \phi_{l}(p) & -\sin \frac{\theta_{l}}{2} \phi_{l}(p) \\
\sin \frac{\theta_{l}}{2} \phi_{l+1}(p) & \cos \frac{\theta_{l}}{2} \phi_{l+1}(p) .
\end{array}\right)
$$

The Hamiltonian can then be written as a $2 N \times 2 N$ matrix with four $N \times N$ blocks,

$$
\begin{gathered}
H_{p \uparrow, p^{\prime} \uparrow}^{R}=\sum_{l} \phi_{l}(p) \phi_{l}^{*}\left(p^{\prime}\right)\left(E_{l+} \cos ^{2} \frac{\theta_{l}}{2}+E_{l-} \sin ^{2} \frac{\theta_{l}}{2}\right), \\
H_{p \uparrow, p^{\prime} \downarrow}^{R}=\sum_{l} \cos \frac{\theta_{l}}{2} \sin \frac{\theta_{l}}{2} \phi_{l}(p) \phi_{l+1}^{*}\left(p^{\prime}\right)\left(E_{l+}-E_{l-}\right),
\end{gathered}
$$

$$
\begin{gathered}
H_{p \downarrow, p^{\prime} \downarrow}^{R}=\sum_{l} \phi_{l+1}(p) \phi_{l+1}^{*}\left(p^{\prime}\right)\left(E_{l+} \sin ^{2} \frac{\theta_{l}}{2}+E_{l-} \cos ^{2} \frac{\theta_{l}}{2}\right), \\
H_{p \downarrow, p^{\prime} \uparrow}^{R}=H_{p^{\prime} \uparrow, p \downarrow}^{R} \dagger^{\dagger} .
\end{gathered}
$$

The spin-flip processes within the ring are included in the off-diagonal parts of $H^{R}$ with respect to the spin orientation; in the absence of the Rashba coupling $\theta_{l}=0$, and both the Hamiltonian and Green's functions become block diagonal. The latter can be computed using the explicit form of the radial functions $\left|\phi_{l}\right\rangle$ and will help us explain the behavior of the spin currents as a function of the magnetic field,

$$
\begin{aligned}
& g_{p \uparrow, p^{\prime} \uparrow}^{R}(E)=\frac{1}{N} \sum_{l} e^{-i\left(\varphi_{p}-\varphi_{p}^{\prime}\right) l}\left(\frac{\cos ^{2} \frac{\theta_{l}}{2}}{E-E_{l,+}+i 0}+\frac{\sin ^{2} \frac{\theta_{l}}{2}}{E-E_{l,-}+i 0}\right), \\
& g_{p \downarrow, p^{\prime} \downarrow}^{R}(E)=\frac{1}{N} \sum_{l} e^{-i\left(\varphi_{p}-\varphi_{p}^{\prime}\right) l}\left(\frac{\sin ^{2} \frac{\theta_{l}}{2}}{E-E_{l,+}+i 0}+\frac{\cos ^{2} \frac{\theta_{l}}{2}}{E-E_{l,-}+i 0}\right) .
\end{aligned}
$$

We remark that both $E_{l,+}$ and $E_{l,-}$ are poles of the Greens' functions and that their weights depend on the tilt angles. At small angles (i.e., for small values of the Rashba strength or large rings), the diagonal elements of the Green's functions with respect to the $\{\uparrow, \downarrow\}$ basis are more sensitive to the poles associated to + states for $g_{p \uparrow, p^{\prime} \uparrow}^{R}$ and - states for $g_{p \downarrow, p^{\prime} \downarrow}^{R}$. It is also important to observe that the exponential terms in Eqs. (12) and (13) depend on the site indices; as we shall see in Sec. III, the matrix elements associated to the contact sites play a major role in transport.

Let us point out that when using lattice models for describing systems with spin-orbit coupling, one introduces a generalized hopping parameter that includes a phase depending on the Rashba parameter (see, e.g., Ref. 13). In our approach, this is not necessary, as we start from the spectral representation (7) in the basis $\{l, s\}$ and then change to the representation $\{p, \sigma\}$.

We now introduce the total Hamiltonian of the coupled system. For simplicity, we consider one-dimensional leads described by a tight-binding Hamiltonian (the leads are semiinfinite). The coupling to the leads is included through a tunneling Hamiltonian containing a smooth time-dependent switching function $\chi(t)$. The role of this function is to ensure that the systems are disconnected at some initial time so that an equilibrium statistical operator can be defined; the steadystate currents are however calculated at some later time when the switching function is time independent and all the transients disappear. For simplicity, we assume that the incident electrons from the leads do not flip their spin when entering the ring and denote by $V^{\alpha}$ the coupling strength to the lead $\alpha$. Each contact implies a pair of sites $\left(0_{\alpha}, p_{\alpha}\right)$, where $p_{\alpha}$ is the site of the ring where the lead is attached, and $0_{\alpha}$ is the 
nearest site of the lead. Then the Hamiltonian reads as (H.c. denotes Hermitian conjugate and $t_{L}$ is the hopping energy on the leads)

$$
\begin{aligned}
H(t)= & \sum_{p, p^{\prime}} \sum_{\sigma, \sigma^{\prime}} H_{p \sigma, p^{\prime} \sigma^{\prime}}^{R}|p \sigma\rangle\left\langle p^{\prime} \sigma^{\prime}\right| \\
& +t_{L} \sum_{\alpha} \sum_{n_{\alpha}, \sigma}\left(\left|n_{\alpha} \sigma\right\rangle\left\langle n_{\alpha}+1, \sigma\right|+\text { H.c. }\right) \\
& +\chi(t) \sum_{\alpha} \sum_{\sigma}\left(V^{\alpha}\left|0_{\alpha} \sigma\right\rangle\left\langle p_{\alpha} \sigma\right|+\text { H.c. }\right) .
\end{aligned}
$$

The steady-state current that enters the lead $\alpha$ is calculated following standard steps within the nonequilibrium Green's function formalism. ${ }^{30}$ In the absence of electron-electron interaction, the lesser and greater Green's functions do not appear in the formula of the current and one only needs the retarded and advanced Green's functions of the coupled ring. They can be computed from the Dyson equation,

$$
G^{R}=g^{R}+g^{R} \sum_{\alpha} \Sigma^{R, \alpha} G^{R}
$$

where $\Sigma^{R}$ is the retarded self-energy of the leads

$$
\Sigma_{p \sigma, p^{\prime} \sigma^{\prime}}^{R, \alpha}(E)=\frac{\left(V^{\alpha}\right)^{2}}{2 t_{L}^{2}} \delta_{p p_{\alpha}} \delta_{p^{\prime} p_{\alpha}} \delta_{\sigma \sigma^{\prime}}\left(E-i \sqrt{4 t_{L}^{2}-E^{2}}\right)
$$

Note that the leads' self-energy contributes with both real and imaginary parts to the pole structure of the effective Green's functions $G^{R, A}$.

The charge current that enters the ring from the lead $\alpha$ is given by

$$
\begin{aligned}
J_{\alpha} & =\frac{e}{h} \int_{-2 t_{L}}^{2 t_{L}} d E \operatorname{Tr}\left\{\Gamma_{\alpha} G^{R} \Gamma_{\beta} G^{A}\left(f_{\alpha}-f_{\beta}\right)\right\} \\
& :=J_{\alpha, \uparrow}+J_{\alpha, \downarrow}
\end{aligned}
$$

The linewidths $\Gamma^{\alpha, \beta}$ are related to the density of states at the end point of the lead $\rho(E)=\sqrt{4 t_{L}^{2}-E^{2}} / \pi(\nu=\alpha, \beta)$,

$$
\Gamma_{p \sigma, p^{\prime} \sigma^{\prime}}^{\nu}(E)=\left(V^{\nu}\right)^{2} \delta_{p p_{\nu}} \delta_{p^{\prime} p_{\nu}} \rho(E)
$$

In the above equations, the trace means a sum over both site indices and spin indices, i.e., $\operatorname{Tr} A=\Sigma_{\sigma=\uparrow, \downarrow} \Sigma_{p}\langle p \sigma|A| p \sigma\rangle$. One can then easily identify the spin currents $J_{\uparrow, \downarrow}$ introduced above. We stress here that the spin currents in the leads are well defined because $\sigma_{z}$ commutes with the Hamiltonian; if we were to compute the currents within the ring, a refined definition would be needed (see, e.g., Ref. 10). It is also easy to see that due to the structure of $\Sigma^{R, \alpha}$, the matrix elements of the Green functions that enter the expression of the currents contain just the contact sites.

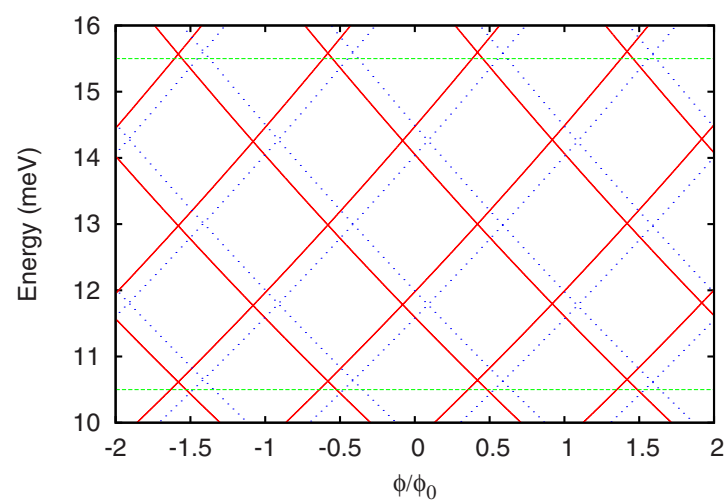

FIG. 2. (Color online) The spectrum of a Rashba ring of radius $R=70 \mathrm{~nm}$ as a function of the magnetic flux. The solid (red) lines represent the eigenvalues $E_{l,+}$ and the dotted (blue) lines are the eigenvalues $E_{l,-}$. The degeneracies between clockwise and counterclockwise states are discussed in the text. We used $N=80$ sites for discretizing the ring, which corresponds to a lattice constant $a=5.49 \mathrm{~nm}$. The Rashba strength is $\alpha=0.5 \times 10^{-11} \mathrm{eVm}$. The dashed lines represent the chemical potentials of the two leads attached to the ring.

The spin filter is characterized by its efficiency

$$
F_{\uparrow, \downarrow}=\frac{J_{\uparrow, \downarrow}}{J_{\uparrow}+J_{\downarrow}} .
$$

Clearly, $F_{\uparrow, \downarrow}$ measures the spin polarization of the current in the leads.

\section{NUMERICAL RESULTS}

Before presenting the numerical results, we shall give some details about the discretization parameters and about the connection to the continuous model. In the definition of the hopping energy of the leads $t_{L}=\hbar^{2} / 2 m^{*} a^{2}$, we use the discretization constant of the ring, that is, $a=2 \pi R / N$. This ensures that the energy of electrons in the leads $\left[-2 t_{L}, 2 t_{L}\right]$ is measured in the same units as the energy levels of the ring. The strength of the Rashba interaction $\alpha_{R}$ lies typically in the range $0.1 \times 10^{-11}-2.5 \times 10^{-11} \mathrm{eVm}$ and we introduce the dimensionless parameter $Q_{R}=(\alpha / R) / \hbar \omega_{0}$ (as usual $h \omega_{0}=\hbar^{2} / 2 m^{*} R^{2}$, and $m^{*}$ is the effective mass of the electron). We take equal coupling to the leads and we introduce the notation $V^{\alpha}=V^{\beta}=\tau$. The number of sites $N$ is a free parameter and determines the number of eigenvalues of the discrete ring. For a fixed ring radius $R$, the agreement between the continuous and discrete spectra improves as $N$ increases.

Since the spectrum of the closed Rashba ring plays a crucial role in understanding the spin interference effects reported below, we shall briefly discuss its properties. Figure 2 shows a few low-energy levels for a ring of radius $R=70 \mathrm{~nm}$ when both Rashba and Zeeman terms are present in the Hamiltonian. The levels are given by analytical expressions both in the discrete and continuous cases. Alternatively, they could be computed by diagonalizing the discrete Hamiltonian described above. It turns out that for $N=80$ sites, the first 25 levels of the ring are in very good agree- 

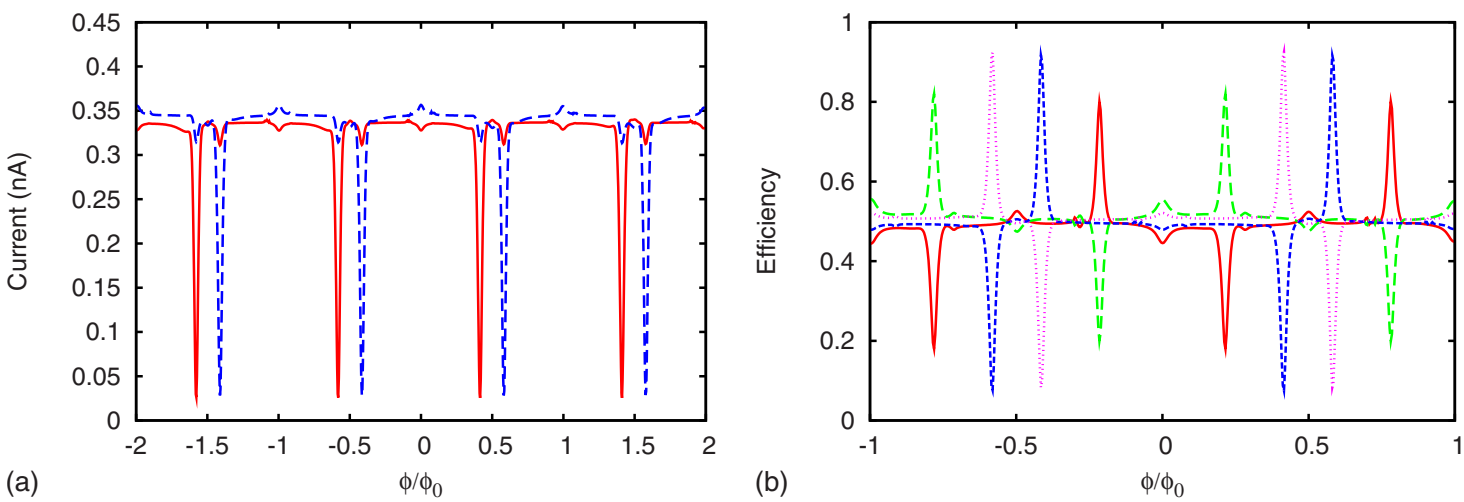

FIG. 3. (Color online) (a) The spin-up (solid line) and spin-down (dashed line) currents for the $70 \mathrm{~nm}$ ring coupled to two leads. The bias is given by $e V=\mu_{\alpha}-\mu_{\beta}$, where $\mu_{\alpha}=15.5 \mathrm{meV}$ and $\mu_{\beta}=10.5 \mathrm{meV}$ (these values are also marked on the spectrum shown in Fig. 2). The Rashba parameter is $\alpha=0.5 \times 10^{-11} \mathrm{eVm}$. (b) The spin filter efficiencies $F_{\uparrow}$ and $F_{\downarrow}$ for two values of the Rashba strength $\alpha=1.0 \times 10^{-11} \mathrm{eVm}\left(F_{\uparrow}\right.$ : solid line; $F_{\downarrow}$ : long-dashed line; $\left.\theta \sim 15^{\circ}\right)$ and $\alpha=0.5 \times 10^{-11} \mathrm{eVm}\left(F_{\uparrow}:\right.$ dashed line; $F_{\downarrow}:$ dotted line; $\left.\theta \sim 22^{\circ}\right)$. By increasing the Rashba strength, the filter efficiency decreases by almost $10 \%$. Other parameters $\tau=0.35, k T=10 \mu \mathrm{eV}$.

ment with the continuous levels and, therefore, we can borrow their analytical form from the continuous case (see, e.g., Ref. 10),

$$
\begin{aligned}
E_{l, \pm}= & \hbar \omega_{0}\left(l-\frac{\Phi}{\Phi_{0}}+\frac{1}{2} \mp \frac{1}{2 \cos \theta_{l}}\right)^{2} \\
& +\frac{\hbar \omega_{0}}{4}\left(1-\frac{1}{\cos ^{2} \theta}\right) \pm \frac{\hbar \omega_{z}}{\cos \theta} .
\end{aligned}
$$

From Fig. 2, we infer that there are particular degeneracy points in the spectrum. First, there is a crossing between clockwise $(\mathrm{CW})$ and counterclockwise $(\mathrm{CCW})$ propagating states with different spin orientation whenever $\Phi_{n}=n \Phi_{0} / 2$. The states with the same spin orientation in the local spin frame but propagating in opposite directions also cross at two values of the flux, which are symmetrically located with respect to $\Phi_{n}$.

The spin filter configuration is realized when two leads with different chemical potentials are attached to the ring. Figure 3(a) presents the spin currents entering the lead $\alpha$ as a function of the magnetic flux. Naturally, in the steady state, the current conservation implies that the charge currents obey the identity $J_{\alpha}=-J_{\beta}$; in all subsequent numerical calculations, this condition is fulfilled. The leads are coupled to opposite sites, more precisely, $p_{\alpha}=N / 2+1$ and $p_{\beta}=1$. We observe the following features: (i) both spin currents exhibit successive sudden drops, which make the spin filtering possible; (ii) the flux values associated to these drops are always around half-integer multiples of flux quanta; (iii) apart from these regions, the two currents are quite comparable and, therefore, a good spin filtering regime is not to be found at any magnetic field. On top of these features, a comparison to the spectrum in Fig. 2 reveals that the sharp minima of the spin currents correspond to degeneracy points between + or between - states that travel clockwise and counterclockwise.

Figure 3(b) shows the spin-up and spin-down efficiencies for two values of the Rashba strength. As expected from Fig. 3(a), $F_{\uparrow}$ and $F_{\downarrow}$ have maxima at the degeneracy points. The increase of $\alpha$ results in larger spacing between the current spikes but also to a reduction in the efficiency.
The above observations suggest that when the filter selects a spin-down (up), the clockwise and counterclockwise $+(-)$ waves interfere destructively in the ring and therefore suppress the spin-up (down) current. This feature is reminiscent of what happens in the ring in the absence of the spinorbit coupling. In that case, one has degeneracies of $\mathrm{CW}$ and CCW states only at $\Phi_{n}=n \Phi_{0} / 2$ [i.e., $E_{l}\left(\Phi_{n}\right)=E_{-l+n}\left(\Phi_{n}\right)$ ]. Then the symmetric location of the leads implies that the exponential term in Eq. (12) reduces to $\cos \pi l$ and that the relevant matrix element of the retarded Green's function $g_{1,(N / 2+1)}^{R}[$ see Eq. (12)] can be rewritten as

$$
\sum_{l}\left(\frac{\cos (\pi l)}{E-E_{l}\left(\Phi_{n}\right)+i 0}-\frac{\cos \pi i(l-n)}{E-E_{-l+n}\left(\Phi_{n}\right)-i 0}\right)
$$

and vanishes when $\Phi=\Phi_{n}$ for any odd $n$. On the other hand, if $n$ is even, the two contributions add to each other. The exact cancellations at $\Phi_{n}$ for $n$ odd simply mean that the clockwise and counterclockwise waves interfere destructively. Our results suggest that the same thing happens in the presence of the Rashba coupling, the difference being that the degeneracy points between $\mathrm{CW}$ and $\mathrm{CCW}$ states with the same spin in the local frame are shifted symmetrically with respect to $\Phi_{n}$. This behavior of the Green's function translates to the Green's functions of the open ring and hence on the spin currents. In Figs. 4(a) and 4(b), we give the transmittances $T_{\sigma \sigma}=\left(e^{2} / h\right)\left|G_{p_{\alpha} \sigma, p_{\beta} \sigma}\right|^{2}$ as a function of magnetic flux and energy $(\sigma=\uparrow, \downarrow)$. The bright traces evidently mimic the + and - parts of the closed ring spectrum (similar plots were reported by Cohen et al. $^{25}$ for molecular rings in the presence of a Zeeman coupling only). Around the degeneracy points, two traces come close to one another. However, at the degeneracy points located near half-integer multiples of $\Phi_{0}$, the transmittances corresponding to $\uparrow$ and $\downarrow$ vanish. This behavior explains the sudden drop of the spin currents at those points since they are roughly obtained by integrating over energy the transmittances. It also supports the discussion about the destructive interference between + or - states around half-integer multiples of $\Phi_{0}$. Another important observation is that the tilt angle determines up to what extent 

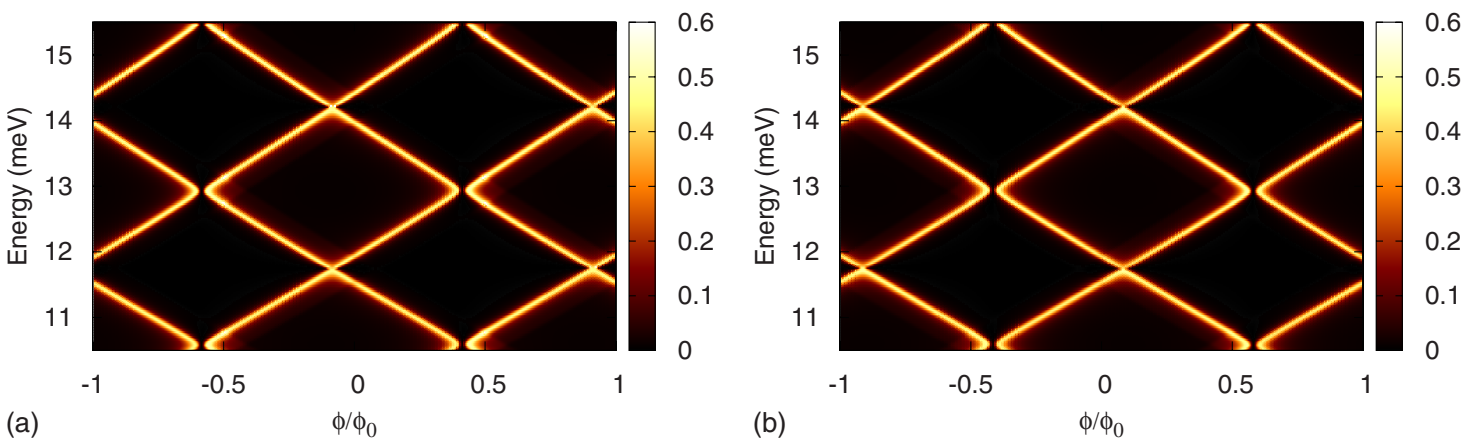

FIG. 4. (Color online) The spin-dependent transmittances as a function of magnetic flux and energy for symmetric coupling to the leads $\delta=45^{\circ}$. (a) $T_{\uparrow, \uparrow}$ and (b) $T_{\downarrow, \downarrow}$. The bias is $e V=\mu_{\alpha}-\mu_{\beta}$, where $\mu_{\alpha}=15.5 \mathrm{meV}$ and $\mu_{\beta}=10.5 \mathrm{meV}$. Other parameters $\tau=0.35$, $\alpha=0.5 \times 10^{-11} \mathrm{eVm}$, and $k T=10 \mu \mathrm{eV}$.

the destructive interference between + states, which is mostly responsible for the drop of $J_{\uparrow}$ also affects $J_{\downarrow}$. From Eqs. (12) and (13), we see that at small $\theta$, one has $\sin \theta \ll 1$ and, therefore, the destructive interference between the + states is not important for the $\downarrow$ component of the Green's function. The situation changes if we increase the Rashba strength: $\theta$ increases and $J_{\downarrow}$ and $J_{\uparrow}$ will both drop. This is why at $\alpha=1.0$ the filter efficiency decreases.

We should point out that a similar suppression of the spin currents at flux values symmetrically located from $\Phi=\Phi_{0} / 2$ was reported by Citro et al. ${ }^{19}$ The authors investigated the spin interference in a ring with a side-coupled dot and focused on a hysteresis effect due to the intradot Cou-

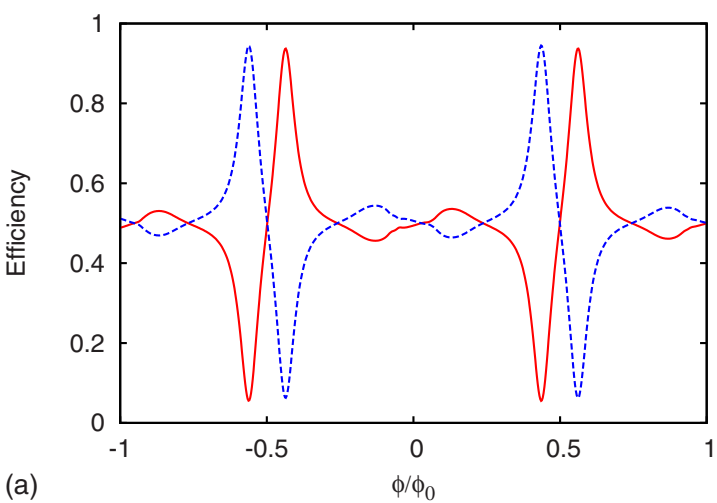

lomb interaction. The connection between the spin filtering and the spectral properties of the ring was not revealed.

We further investigate the efficiency of spin filtering for rings of different size, while keeping the Rashba coupling fixed (i.e., $\alpha=0.5 \times 10^{-11} \mathrm{eVm}$ ). We present in Figs. 5(a) -5 (c) results for $R=60,90$, and $120 \mathrm{~nm}$ rings. The spin filter efficiency decreases to $80 \%$ for the larger ring, but the advantage is that the separation between the points of destructive interference is better. This could be crucial in experiments where it would be difficult to observe spin filtering by tuning the magnetic field by a few mT only. For the 60 nm ring, we find that the difference between the magnetic fields corresponding to the two minima located around

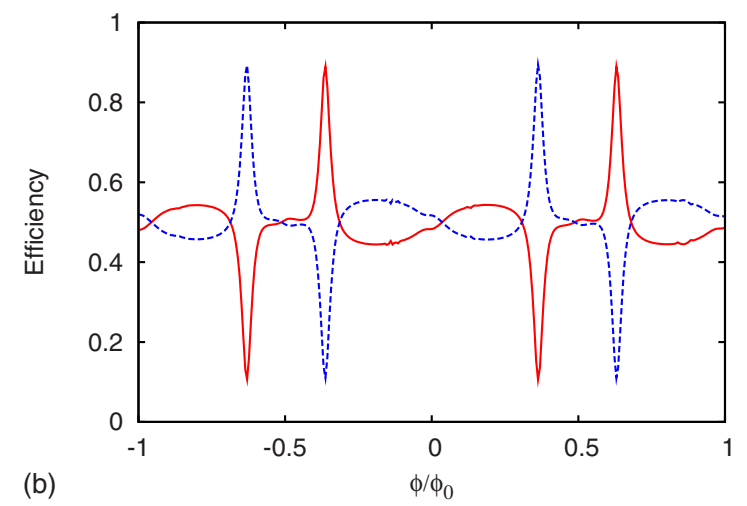

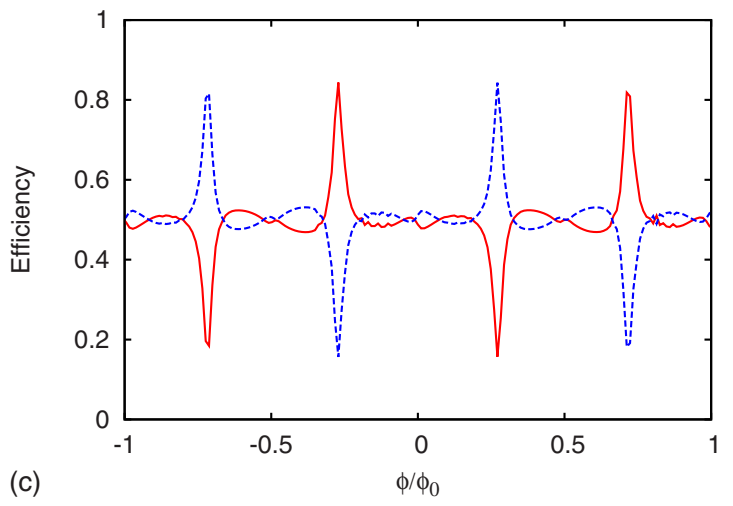

FIG. 5. (Color online) The spin filter efficiencies $F_{\uparrow}$ (solid line) $F_{\downarrow}$ (dashed line) as a function of the magnetic flux for rings of different radii (a) $R=60 \mathrm{~nm}$ ring, (b) $R=90 \mathrm{~nm}$, and (c) $R=120 \mathrm{~nm}$. The Rashba coupling $\alpha=0.5 \times 10^{-11} \mathrm{eVm}, \tau=0.5, \mu_{\alpha}=15.51 \mathrm{meV}$, and $\mu_{\beta}=10.5 \mathrm{meV}$. 

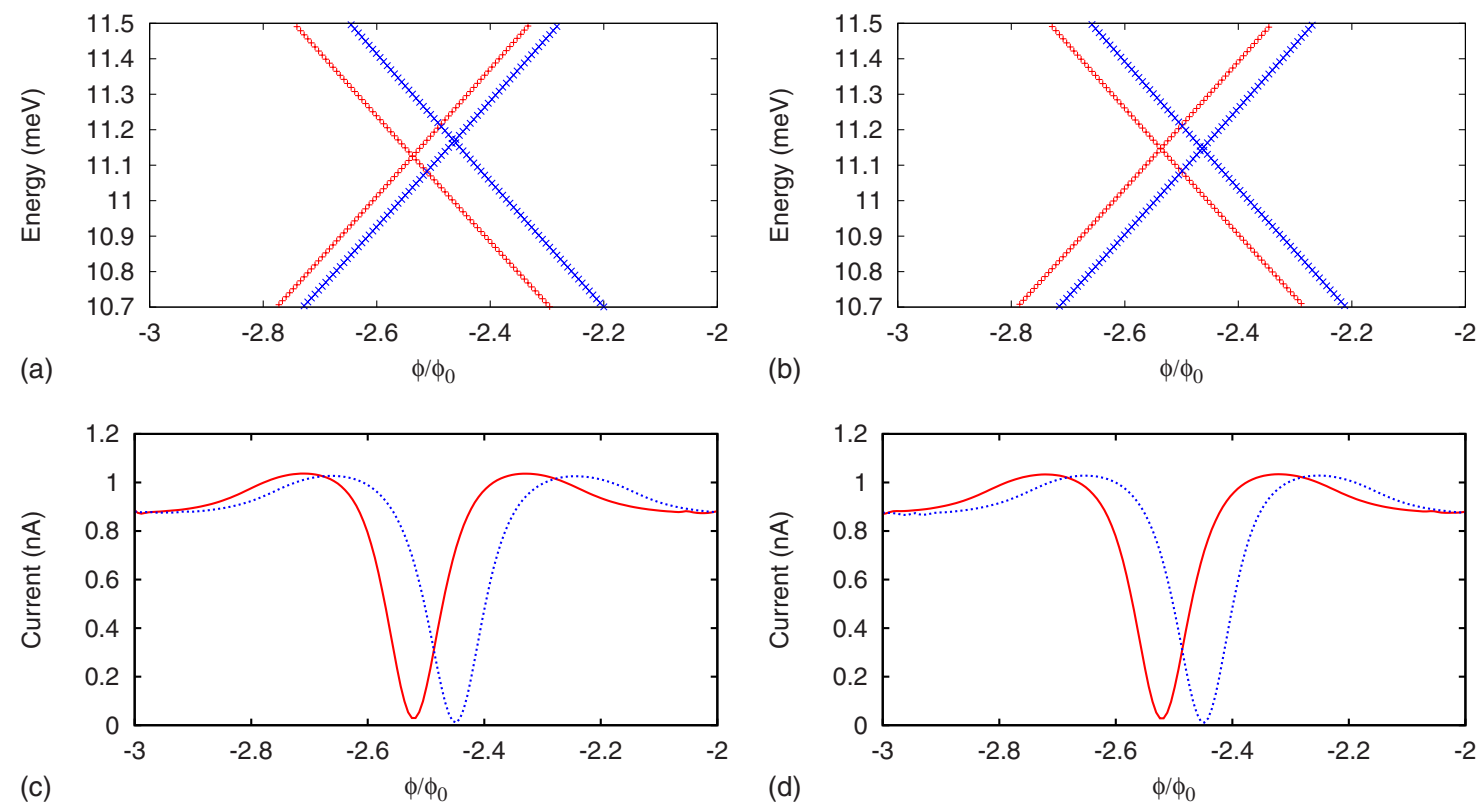

FIG. 6. (Color online) (a) A crossing region from the spectrum of a ring having $R=90 \mathrm{~nm}$ in the presence of the Zeeman term. (b) The same region when the Zeeman term is disregarded in the calculation. We remark that the Zeeman term destroys the symmetry of the spectrum with respect to $\phi=2.5 \phi_{0}$. (c) The spin currents as a function of the magnetic flux when the Zeeman term is considered (spin up: solid line; spin down: dashed line). (d) The same, in the absence of the Zeeman term. Other parameters: $\tau=0.75, \alpha=0.5 \times 10^{-11} \mathrm{eVm}$, and $k T=10 \mu \mathrm{eV}$.

$\phi=0.5$ is $\Delta B=3.5 \mathrm{mT}$ while for the $90 \mathrm{~nm}$ ring $\Delta B=22 \mathrm{mT}$.

As we have stated already, the Zeeman term is included in our numerical simulations. This term can be neglected in the so-called adiabatic regime when $\omega_{z} \ll \omega_{0}$. However, for a ring with $R=90 \mathrm{~nm}$ it turns out that if $\phi \in\left(2 \phi_{0}, 3 \phi_{0}\right)$ the magnetic field ranges from 0.24 to $0.155 \mathrm{~T}$, and in this range one has $\omega_{0} / \omega_{z} \sim 2.3$. Then we selected a region from the spectrum of the ring, where the energies of the clockwise and counterclokwise spin $+/-$ states experience various crossings. In Figs. 6(a) and 6(b), we present these crossings with and without the Zeeman term. The effect of the Zeeman term is evident: it twists and shrinks the rhomboidal configuration of the four crossing points seen in the absence of the Zeeman term (note that it this case, there are two degeneracy points at $\left.\phi=2.5 \phi_{0}\right)$. However, the effect of the Zeeman term on the spectrum does not significantly alter the spin filtering. The spin currents computed with and/without the Zeeman term shown in Figs. 6(c) and 6(d), respectively, are rather similar - the only difference is that in the absence of the Zeeman term, a mirror symmetry with respect to $\phi=2.5 \phi_{0}$ is noticed.

The next step in our analysis refers to the robustness of the spin filter (i.e., of its efficiency) with respect to the variations in the bias. To this end, we fixed the magnetic flux to a value, which corresponds to a degeneracy point for the states in the ring and we varied the chemical potential of the left lead. The results for rings of radii $R=60,70$, and $90 \mathrm{~nm}$ are shown in Fig. 7. It is obvious that in all three cases, the spin-up currents increase steplike as more levels of the ring enter the bias window, while the spin-down currents remain much smaller. Thus, the destructive interference between states guarantees a good filter efficiency even at a finite bias.
For rings of 70 and $90 \mathrm{~nm}$, the spectrum is more dense, leading to a large number of steps in the spin-up currents.

In the numerical simulations, we have used the same number of sites $N=120$ for the three rings, which means that the lattice constant $a=2 \pi R / N$ increases with the ring radius. In turn, this implies that the currents measured in units of $e t_{L} / \hbar$ decrease when the ring radius increases since $t_{L}=\hbar^{2} / 2 m^{*} a^{2}$.

We have also performed numerical simulations for other temperatures and found that while the currents are affected by the increase in the temperature the filter efficiency is still very good.

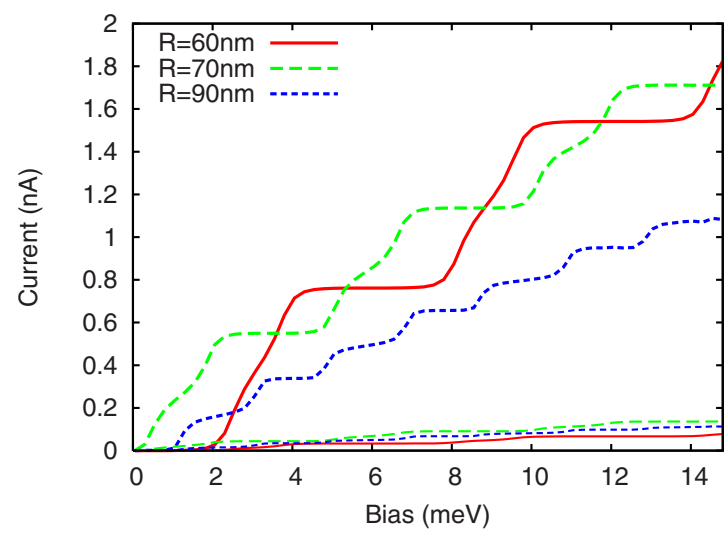

FIG. 7. (Color online) The spin-up (steplike increasing curves) and spin-down currents (slowly increasing curves at the bottom) in the right lead as a function of bias $e V=\mu_{\alpha}-\mu_{\beta}$ for rings of different radii. We keep $\mu_{\beta}=0.0105$ and vary $\mu_{\alpha}$. The magnetic fluxes are chosen that the states in the ring interfere destructively so that the spin-down currents are almost suppressed. Other parameters: $\tau=0.5, \alpha=0.5 \times 10^{-11} \mathrm{eVm}$, and $k T=10 \mu \mathrm{eV}$. 


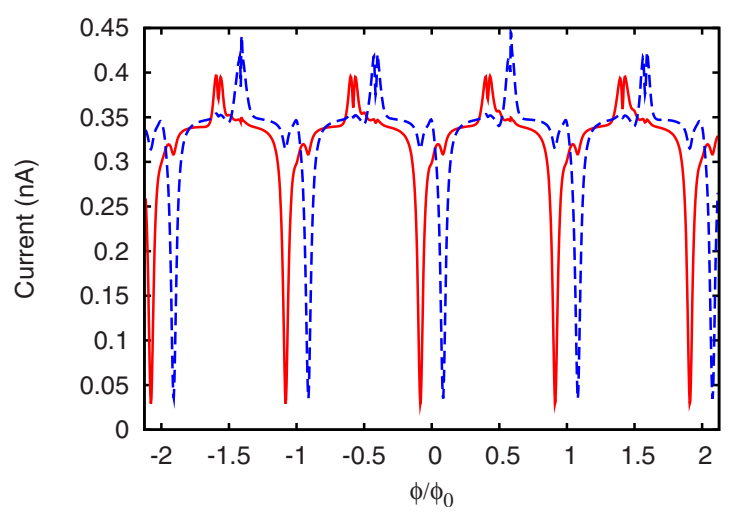

FIG. 8. (Color online) The spin-up (solid line) and spin-down (dashed line) currents entering the left lead as a function of the magnetic flux in the asymmetric configuration $p_{\alpha}=N / 2+1$ and $p_{\beta}$ $=N / 8$. Other parameters: $R=70 \mathrm{~nm}, \tau=0.35, \alpha=0.5 \times 10^{-11} \mathrm{eVm}$, and $k T=10 \mu \mathrm{eV}$.

Our previous analysis of the Green's functions for both closed and open rings was done for the rather particular case of symmetric coupling, i.e., $p_{\alpha}-p_{\beta}=N / 2$. What happens if we change the right contact by taking $p_{\beta}=N / 8$ ? The asymmetry can be conveniently described by the angle $\delta$ between the two leads (in this case, the angle between the two leads is now $\left.135^{\circ}\right)$ ? An asymmetric setup of the leads means that the clockwise and counterclockwise spin states acquire different $\mathrm{AB}$ and $\mathrm{AC}$ phases while propagating toward the right lead because the paths they experience are of different length. This will presumably alter their interference at the contact point and, hence, the outgoing charge and spin currents. From Fig. 8, we see that the ring still filters up or down spins, but a comparison with Fig. 3(a) reveals a striking difference: the flux values at which the filter operation is effective are symmetrically located to the left and right sides of the integer multiples of flux quanta instead of half-integer as before.

We show in Figs. 9(a) and 9(b) the corresponding transmittances for the coupled ring. One should notice the presence of minima around integer multiples of $\Phi_{0}$ and their correspondence to the sharp drops of the spin-up and spindown currents.

We have also analyzed the oscillations of the charge current as a function of the dimensionless parameter $Q_{R}$ defined at the beginning of this section. Figure 10 shows the results

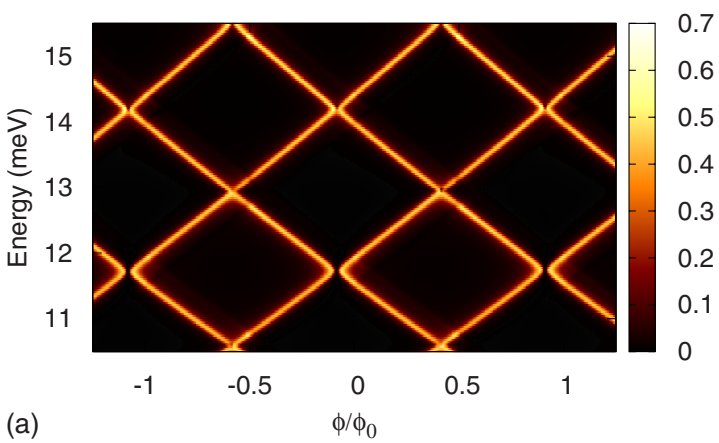

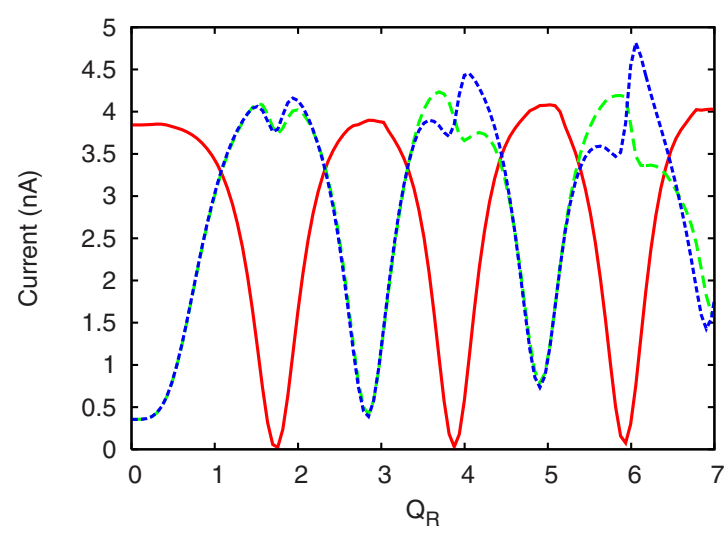

FIG. 10. (Color online) The quasiperiodic oscillations of the charge current as a function of the parameter $Q_{R}$, for symmetric and asymmetric couplings to the leads. Solid line: symmetric coupling $\delta=180^{\circ}$; dashed line: $\delta=45^{\circ}$; dotted line: $\delta=315^{\circ}$. Other parameters: $R=70 \mathrm{~nm}, \tau=1.0$, and $k T=10 \mu \mathrm{eV}$.

at vanishing magnetic fields. In the case of symmetric coupling, the result is in full agreement with the previous conductance calculations by Frustaglia and Richter; ${ }^{11}$ one should note that the oscillation period in our case in also larger that unity, as was reported in Ref. 7. This difference is due to the fact that in Ref. 7, it is assumed that the spinors of the ring are aligned with the effective magnetic field given by the Rashba coupling. We also show oscillations for two asymmetric coupling configurations, i.e., $p_{\beta}=N / 8$ and $p_{\beta}=7 N / 8$. The differences are rather striking: the previous maxima turn to minima in both asymmetric configurations and we also observe twin local maxima. Each such doublet has different amplitudes, the difference increasing with the Rashba strength. The asymmetry of the doublet is reversed by changing the angle between the leads from $45^{\circ}$ to $315^{\circ}$. Note however that the period of the oscillations does not change.

This behavior of the current suggests that, similar to what we observed when the magnetic flux varies, the nature of the interference within the ring also changes in the asymmetric configuration when the Rashba coupling is changed. Note also that even at $\alpha=0$, the symmetric configuration leads to maxima in the current, while in the asymmetric configuration minima are found at the same location. The different amplitudes of the twin local maxima (in the asymmetric case) could be due to separate interference between spin + and

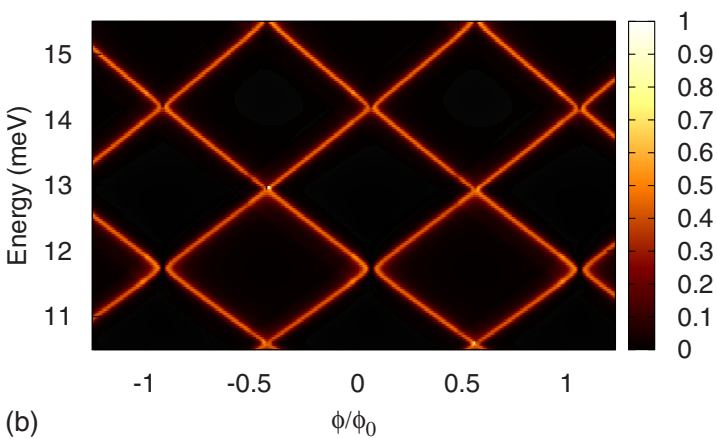

FIG. 9. (Color online) The spin-dependent transmittances as a function of magnetic flux and energy for asymmetric coupling to the leads $\delta=45^{\circ}$. (a) $T_{\uparrow}$ and (b) $T_{\downarrow}$. Other parameters: $R=70 \mathrm{~nm}, \tau=0.35, \alpha=0.5 \times 10^{-11} \mathrm{eVm}$, and $k T=10 \mu \mathrm{eV}$. 
spin - states that are separated in the presence of the Rashba coupling.

\section{CONCLUSIONS AND OPEN PROBLEMS}

In this work, we have presented theoretical calculations for the electronic transport in a Rashba interferometer subjected to a perpendicular magnetic field and coupled to two leads in various geometries. The spin currents were computed using the nonequilibrium Green's functions (Keldysh) formalism. By analyzing the spectrum of the closed ring and the relevant Green's functions of the open ring, we were able to identify a class of optimal parameters for the spin filter operation (e.g., special values of the magnetic field). We have emphasized the connection between a good efficiency of the spin filter and the degeneracy points between different spin states within the ring. The symmetric coupling to the leads is an optimal configuration for spin filtering. Our numerical results show that even at a finite bias and moderate
Rashba strength, one should get up to $95 \%$ spin-polarized currents in the leads. The oscillations of the current as a function of the Rashba strength are analyzed as well both for symmetric and asymmetric configurations. We show that an asymmetric coupling to the leads turns maxima into minima and induces double peak structures in the current oscillations. Here, we have considered uniform Rashba strength along the wire and performed calculations for electronic transport. However, one could also investigate the effect of an inhomogeneous Rashba coupling for both holes and electrons. ${ }^{15,31}$

\section{ACKNOWLEDGMENTS}

This work is supported by TUBITAK (Grant No. 108T743) and TUBA. V.M. acknowledges the hospitality of the Bilkent University, where this work was initiated, and the financial support from PNCDI2 program under Grants No. 515/2009 and No. 45N/2009 and TUBITAK-BIDEP.
${ }^{1}$ S. Washburn and R. A. Webb, Adv. Phys. 35, 375 (1986).

${ }^{2}$ M. Kohda, T. Bergsten, and J. Nitta, J. Phys. Soc. Jpn. 77, 031008 (2008); J. Nitta and T. Bergsten, New J. Phys. 9, 341 (2007).

${ }^{3}$ G. Engels, J. Lange, Th. Schapers, and H. Luth, Phys. Rev. B 55, R1958 (1997).

${ }^{4}$ C.-M. Hu, J. Nitta, T. Akazaki, H. Takayanagai, J. Osaka, P. Pfeffer, and W. Zawadzki, Phys. Rev. B 60, 7736 (1999).

${ }^{5}$ D. Grundler, Phys. Rev. Lett. 84, 6074 (2000).

${ }^{6}$ E. I. Rashba, Fiz. Tverd. Tela (Leningrad) 2, 1224 (1960) [Sov. Phys. Solid State 2, 1109 (1960)].

${ }^{7}$ J. Nitta, F. E. Meijer, and H. Takayanagi, Appl. Phys. Lett. 75, 695 (1999).

${ }^{8}$ T. Bergsten, T. Kobayashi, Y. Sekine, and J. Nitta, Phys. Rev. Lett. 97, 196803 (2006).

${ }^{9}$ J. Nitta, T. Bergsten, Y. Kunihashi, and M. Kohda, J. Appl. Phys. 105, 122402 (2009).

${ }^{10}$ J. Splettstoesser, M. Governale, and U. Zülicke, Phys. Rev. B 68, 165341 (2003).

${ }^{11}$ D. Frustaglia and K. Richter, Phys. Rev. B 69, 235310 (2004).

${ }^{12}$ B. Molnár, F. M. Peeters, and P. Vasilopoulos, Phys. Rev. B 69, 155335 (2004).

${ }^{13}$ S. Souma and B. K. Nikolić, Phys. Rev. B 70, 195346 (2004).

${ }^{14}$ B. Nikolić, L. Zarbo, and S. Souma, in The Oxford Handbook on Nanoscience and Technology: Frontiers and Advances, edited by A. V. Narlikar and Y. Y. Fu (Oxford University Press, Oxford, 2010), Vol. I, Chap. 24, pp. 814-866.

${ }^{15}$ M. F. Borunda, X. Liu, A. A. Kovalev, X.-J. Liu, T. Jungwirth, and J. Sinova, Phys. Rev. B 78, 245315 (2008).
${ }^{16}$ D. Stepanenko, M. Lee, G. Burkard, and D. Loss, Phys. Rev. B 79, 235301 (2009).

${ }^{17}$ P. Földi, O. Kálmán, M. G. Benedict, and F. M. Peeters, Phys. Rev. B 73, 155325 (2006)

${ }^{18}$ R. Citro, F. Romeo, and M. Marinaro, Phys. Rev. B 74, 115329 (2006).

${ }^{19}$ R. Citro and F. Romeo, Phys. Rev. B 77, 193309 (2008).

${ }^{20}$ P. Vasilopoulos, O. Kálmán, F. M. Peeters, and M. G. Benedict, Phys. Rev. B 75, 035304 (2007).

${ }^{21}$ P. Lucignano, D. Giuliano, and A. Tagliacozzo, Phys. Rev. B 76, 045324 (2007).

${ }^{22}$ Z. Zhu, Y. Wang, K. Xia, X. C. Xie, and Z. Ma, Phys. Rev. B 76, 125311 (2007).

${ }^{23}$ R. Capozza, D. Giuliano, P. Lucignano, and A. Tagliacozzo, Phys. Rev. Lett. 95, 226803 (2005).

${ }^{24}$ S. Bellucci and P. Onorato, J. Phys.: Condens. Matter 19, 395020 (2007).

${ }^{25}$ G. Cohen, O. Hod, and E. Rabani, Phys. Rev. B 76, 235120 (2007).

${ }^{26}$ U. Aeberhard, K. Wakabayashi, and M. Sigrist, Phys. Rev. B 72, 075328 (2005).

${ }^{27}$ F. E. Meijer, A. F. Morpurgo, and T. M. Klapwijk, Phys. Rev. B 66, 033107 (2002).

${ }^{28}$ M. Lee and C. Bruder, Phys. Rev. B 73, 085315 (2006).

${ }^{29}$ K.-W. Chen and C.-R. Chang, Phys. Rev. B 78, 235319 (2008).

${ }^{30}$ H. Haug and A.-P. Jauho, Quantum Kinetics in Transport and Optics of Semiconductors (Springer-Verlag, Berlin, 1996).

${ }^{31}$ Y. Tserkovnyak and A. Brataas, Phys. Rev. B 76, 155326 (2007). 